\title{
AUF DER SUCHE NACH DEM ANDEREN IN DER WIEDERKEHR DES GLEICHEN. HEINER MÜLLERS BILDBESCHREIBUNG ZWISCHEN POST-HISTORISCHEN ENDZEITFANTASIEN UND HOFFNUNG AUF GESCHICHTE AM ENDE DES KALTEN KRIEGES
}

\begin{abstract}
Looking for the other in the return of the same. Heiner Müller's Bildbeschreibung, astride the fantasized end of history and the hope of a new historical dynamism towards the end of the Cold War

Bildbeschreibung, written in 1984, is undoubtedly the most hermetic text in the work of the EastGerman dramatist Heiner Müller. In this prose text, referred to by its author as 'a play', an ungraspable "I" vainly attempts to piece together an image to construct a coherent whole. When one compares this with Müller's other works, Bildbeschreibung makes disconcerting reading because of its apparent lack of any political dimension. A thorough analysis however reveals that the traumas of Germany's twentieth century history underpin the narrative. Moreover, the desperate attempts by the "I" to build itself into a proper "self" through the (re)-construction of the undecipherable image, hark back to the difficulties one has to conceptualize History after the end of what Jean-François Lyotard calls "the grand narratives'. Bildbeschreibung stages these difficulties in terms of history as drama. The "landscape between steppe and savannah" under "a Prussian blue sky" turns out to be the GDR in the 1980 s, a country tossed and torn between the anguish of the ending of the historical process as described by the theories of post-history and the hope of a new dynamism made possible by the end of the Cold War.
\end{abstract}

„Ich habe mich aus biographischen oder aus geographischen Gründen immer für Geschichte interessiert, oder mich interessieren müssen für sie." ${ }^{11}$ Von den ersten dramatischen Versuchen unmittelbar nach dem Zweiten Weltkrieg bis zu seinem letzten, unvollendet gebliebenen Theaterstück ${ }^{2}$ hat sich der ostdeutsche Dramatiker

\footnotetext{
${ }^{1}$ Heiner Müller: Für ein Theater, das an Geschichte glaubt, Gespräch mit Flavia Foradini (1988), in: ders.: Werke, Frankfurt a.M., Bd. 11: Gespräche 2 1987-1991, 2008 (=W11), S. 332-340, Zitat S. 333.

${ }^{2}$ Heiner Müller: Germania 3 Gespenster am toten Mann (veröffentlicht postum 1996), in: ders.: Werke, Bd. 5: Die Stücke 3, 2002 (= W5), S. 253-296.
} 
Heiner Müller geradezu obsessiv mit der deutschen Geschichte und mit Geschichte überhaupt befasst. Diese erscheint in Müllers Texten stets, ganz im Einklang mit der marxistischen Vorstellung, als eine Abfolge von gesellschaftlichen Kämpfen und Widersprüchen. Es mag deshalb erstaunen, wenn die 1984 entstandene Bildbeschreibung ${ }^{3}$ in Zusammenhang mit dem Posthistoire gebracht wird, einem Begriff aus dem Umkreis des postmodernen Denkens. Zwar ziehen Müllers Texte das Leitbild eines teleologisch gesteuerten Gangs der Geschichte in Richtung einer kommunistischen Gesellschaft immer stärker in Zweifel, doch haben sie nichts gemein mit dem unbekümmerten Spiel mit den Trümmern der großen Denksysteme der Vergangenheit, als das sich die Postmoderne gern präsentiert. Versuche, den Autor dort anzusiedeln ${ }^{4}$, haben deshalb auch kaum überzeugen können. Nun scheint mit Bildbeschreibung ein Sonderfall vorzuliegen, der in Müllers Werk nur schwer zu verorten ist, da keinerlei politischen Inhalte mehr in ihm auszumachen sind. Der Autor selbst bestätigt dies, wenn er in seiner Autobiographie die Arbeit an diesem Text als „Urlaub von der DDR“, als „ein[en], vielleicht narzisstische[n], Befreiungsakt" bezeichnet. $^{5}$ Der vorliegende Artikel möchte dem entgegenhalten, dass erstens Heiner Müllers Theaterstücke durchaus als Entwicklung auf Bildbeschreibung hin gelesen werden können, dass zweitens im Zentrum dieses Texts sehr wohl die Gespenster der deutschen Geschichte stehen und dass drittens Müller hier versucht, in einer historischen Umbruchsituation - dem Ende des Kalten Kriegs Mitte der 1980er Jahre - seine Vorstellungen von Geschichte neu zu ordnen: zwischen der Angst vor dem definitiven Steckenbleiben des historischen Prozesses und der Hoffnung auf eine neue geschichtliche Dynamik.

\section{Weg von der Geschichte mit „Hand und Fuß“: Heiner Müllers Texte bis Bildbeschreibung}

Müllers erstes veröffentlichtes Theaterstück, Der Lohndrücker $(1957)^{6}$, beschreibt mit einer bislang nie dagewesenen Offenheit die Widersprüche der jungen DDR: Wie kann die neue Gesellschaft aufgebaut werden mit Menschen, die noch von ihrer nationalsozialistischen Vergangenheit geprägt sind? Und wie kann eine Gesellschaftsordnung, die den Menschen doch die Erfüllung ihres ureigenen

\footnotetext{
${ }^{3}$ In: Heiner Müller: Werke, Bd. 2: Die Prosa, 1999 (=W2), S. 112-119.

${ }^{4}$ Siehe dazu: Arlene Akiko Teraoka: The Silence of Entropy or Universal Discourse. The Postmodernist Poetics of Heiner Müller, New York/Bern/Frankfurt a.M. 1985.

${ }^{5}$ Heiner Müller: Krieg ohne Schlacht. Leben in zwei Diktaturen (1992), in: ders.: Werke, Bd. 9: Eine Autobiographie (=W9), 2005, S. 7-291, Zitat S. 269.

${ }^{6}$ In: Heiner Müller: Werke, Bd. 3: Die Stücke 1, 2000 (=W3), S. 27-64. Die Jahreszahl in Klammern bezeichnet jeweils das Jahr der Fertigstellung des Werks.
} 
Glücksanspruchs verspricht, von ihnen neuerliche Opfer verlangen? Der Autor nimmt dabei ein Projekt Brechts auf, das dieser aufgegeben hatte; zu unbequem war das Thema nach dem 17. Juni 1953 geworden. Müller hingegen lässt sich nicht abschrecken, erregt auch mit dem folgenden Stück Die Korrektur (1. Fassung 1957 2. Fassung $1958^{8}$ ) Aufmerksamkeit, eckt an, wird dennoch als junges Talent gelobt und mit dem Heinrich-Mann-Preis ausgezeichnet. Erst der Skandal nach der Uraufführung der Komödie Die Umsiedlerin oder Das Leben auf dem Lande $(1961)^{9} \mathrm{im}$ Kontext des Mauerbaus führt zur Zäsur: Der Autor wird aus dem Schriftstellerverband der DDR ausgeschlossen, was einem Berufsverbot gleichkommt, und schreibt über ein Jahrzehnt lang für die sprichwörtliche Schublade. In dieser Zeit arbeitet er an ganz verschiedenen Theatertexten: Bearbeitungen mythologischer Stoffe wie Philoktet (1964) $)^{10}$, Herakles $5(1966)^{11}$ und Prometheus $(1968)^{12}$; Dramen, die ungeschminkt die Widersprüche der Revolution behandeln, etwa Mauser $(1970)^{13}$ und Zement (1972) ${ }^{14}$; schließlich Stücke, die um die deutsche Geschichte und ihre Gespenster kreisen und von denen insbesondere Germania Tod in Berlin (1971) ${ }^{15}$, Die Schlacht $(1974)^{16}$, und Leben Gundlings Friedrich von Preußen Lessings Schlaf Traum Schrei $(1976)^{17}$ hervorzuheben sind. Inzwischen ist Zement unter der Leitung von Ruth Berghaus 1973 am Berliner Ensemble aufgeführt worden, was die Rückkehr Müllers ins Theaterleben der DDR und eine halbe Rehabilitierung bedeutet; ab 1974 erscheinen seine Texte im westdeutschen Rotbuch-Verlag, ein erstes Anzeichen für die Aufmerksamkeit, die der Autor im bundesrepublikanischen Literaturbetrieb mehr und mehr erregen wird.

Versuche, Heiner Müller in den Griff zu bekommen, indem man ihn in Schubladen - „Antiken-Müller“, „Revolutions-Müller“ und „Deutschland-Müller“ - einsortiert und die verschiedenen Werkgruppen in eine chronologische Abfolge bringt, sind jedoch zum Scheitern verurteilt: $\mathrm{Zu}$ sehr sind die verschiedenen Themen ineinander verschränkt, zu lange arbeitet der Autor an den einzelnen Texten, manchmal über Jahrzehnte. Eines lässt sich jedoch feststellen: Ist bereits Der Lohndrücker von geradezu lakonischer Kürze und zeichnen sich seine einzelnen Szenen durch große Eigenständigkeit aus, so verstärkt sich der Hang zu Konzentration und Fragmentie-

\footnotetext{
${ }^{7}$ In: W3, S. 109-126.

${ }^{8}$ In: $W 3$, S. $127-146$

${ }^{9}$ In: W3, S. 181-287.

${ }^{10}$ In: $W 3$, S. $289-327$.

${ }^{11}$ In: $W 3$, S. 397-409.

${ }^{12}$ In: Heiner Müller, Werke, Bd. 4: Die Stücke 2, 2001 (=W4), S. 7-45.

${ }^{13}$ In: W4, S. 243-260.

${ }^{14}$ In: W4, S. 379-467.

${ }^{15}$ In: W4, S. 325-377.

${ }^{16}$ In: W4, S. 469-482.

${ }^{17}$ In: $W 4$, S. $509-537$.
} 
rung von Stück zu Stück. Einen ersten Höhepunkt erreicht diese Tendenz mit Die Hamletmaschine $(1977)^{18}$. Ursprünglich geplant als Übersetzung von Shakespeares Drama, reduziert sich Müllers Text auf schließlich knapp zehn Seiten und fünf Szenen, in denen Monolog und stummes Spiel vorherrschen. Und doch ist hier auf kleinstem Raum der ganze Heiner Müller enthalten: Hamlet wird zur Figur des Intellektuellen, der nicht weiß, wie er sich in Zeiten revolutionärer Umbrüche verhalten soll; durch die Intrige von Helsingör scheinen der 17. Juni 1953 und der Ungarnaufstand von 1956 durch - nicht umsonst lautet der Arbeitstitel des Stücks HiB: Hamlet in Budapest. ${ }^{19}$ Der Bericht über die gescheiterte Uraufführung der Hamletmaschine 1977 in Köln - an eine Aufführung in der DDR ist zu dieser Zeit nicht zu denken begründet im Westen den Mythos von Heiner Müller als einem hermetischen, tendenziell unspielbaren Dichter ${ }^{20}$ und bestärkt die Betonköpfe auf der anderen Seite der Mauer, die den Autor bereits nach dem Umsiedlerin-Skandal als „Beckett des Ostens" beschimpft hatten ${ }^{21}$, in ihren Vorurteilen. Richtig an dieser Einschätzung ist jedoch, dass Müllers Texte dem Theater erheblichen Widerstand entgegensetzen. Nicht nur erzeugt die Verknappung der Sprache Lücken, die durch die Inszenierung und durch den mitdenkenden Zuschauer geschlossen werden müssen. Die immer prekärer werdende Interaktion, für die die Tendenz zum Monolog in der Hamletmaschine ein gutes Beispiel ist, verlagert die eigentliche Handlung ins Innere der einzelnen Figuren, die schließlich „das Schlachtfeld in der Brust ${ }^{، 22}$ tragen. Es ist nur folgerichtig, wenn der Hamletdarsteller von sich sagt:

Mein Platz, wenn mein Drama noch stattfinden würde, wäre auf beiden Seiten der Front, zwischen den Fronten, darüber. [...] Meine Rollen sind Speichel und Spucknapf Messer und Wunde Zahn und Gurgel Hals und Strick. ${ }^{23}$

Zum Austragungsort der Konflikte wird dabei aber nicht nur die Person auf der Bühne, sondern auch, und vor allem, der Zuschauer, der die Konflikte aufgebürdet bekommt und mit sich selbst ausmachen muss. Keine Geschichte, auch keine Fabel im brechtschen Sinne, präsentiert ihm eine möglichen Lösung. In einem Brief an den Theaterkritiker Martin Linzer kommentiert der Autor sein Vorgehen folgendermaßen: „Ich glaube nicht, dass eine Geschichte, die ,Hand und Fuß` hat (die Fabel im

\footnotetext{
${ }^{18}$ In: W4: S. $543-554$.

${ }^{19}$ Siehe dazu: Heiner Müller: Manuscrits de Hamlet-Machine, transcription de Julia Bernhard, traduction de Jean Jourdheuil et Heinz Schwarzinger, Paris 2003.

${ }^{20}$ Die Hamletmaschine. Heiner Müllers Endspiel, hrsg. von Theo Girshausen, Köln 1978.

${ }^{21}$ So Siegfried Wagner, Chef der Abteilung Kultur im ZK der SED, auf der Versammlung des Schriftstellerverbands der DDR vom 17.10.1961. Zitiert nach: Heiner Müller: Krieg ohne Schlacht, in: W9, S. 135.

${ }^{22}$ Heiner Müller: Traktor, in: W4, S. 483-504, Zitat S. 503.

${ }^{23}$ W4, S. $550 \mathrm{f}$.
} 
klassischen Sinn), der Wirklichkeit noch beikommt. “24 Dieser Angriff auf Ganzheit und Unversehrtheit findet sich auf allen Ebenen: Der Traktorist in Müllers Agrodrama, der beim Pflügen auf eine Mine fährt und durch die Explosion ein Bein verliert ${ }^{25}$, der stumme Protagonist der Szene „Nachtstück“ in Germania Tod, der sich aus Verzweiflung Arme und Beine ausreißt ${ }^{26}$, die Kleistfigur in Leben Gundlings, die Puppen seziert ${ }^{27}$, sie alle führen den Vorgang der Amputation auf der inhaltlichen Ebene vor. Dem Text selbst ergeht es dabei keineswegs besser: Der Autor kappt Sinnzusammenhänge, indem er zwischen einzelne Szenen Texte einschiebt, die manchmal als Zitate und Selbstzitate kenntlich sind, manchmal die Funktion eines Autorenkommentars einnehmen, öfter noch an kafkasche Parabeln gemahnen und deren Zusammenhang mit dem Ganzen in jedem Fall mühsam rekonstruiert, wenn nicht gar überhaupt erst konstruiert werden muss. Nicht zufällig findet sich unter den in Traktor eingefügten Texten ein Auszug aus dem Mythos des Vorsokratikers Empedokles von der Entstehung des Menschen: Im Gegensatz zu Platons Gastmahl wird hier nicht von einer ursprünglichen und dann verloren gegangenen Einheit erzählt ${ }^{28}$, sondern von einzelnen Gliedmaßen, die der Erde entsteigen, sich zufällig zusammenfügen und nur dann, wenn die Vereinigung zu einem sinnvollen Ergebnis führt, dauerhaft überleben. ${ }^{29}$ Das gesamte Werk Heiner Müllers handelt in der einen oder anderen Weise vom Ende der Erzählung als Garanten des einen, unumstrittenen Sinns. An deren Stelle tritt der Text als Textum ineinander verwobener und sich durchkreuzender Sinnzusammenhänge. Wobei die Story natürlich auch auf die Historie verweist: Immer wieder finden sich bei Müller Verweise auf den Philosophen Walter Benjamin, der Geschichte nicht als kontinuierlichen Prozess, sondern als Folge von Niederlagen und Brüchen zu erfassen sucht. ${ }^{30}$ Literarisch umgesetzt wird dies nicht zuletzt durch die Aneinanderreihung von Inkommensurablem, eine Parataxe, die sich bis in die Titel der Stücke hinein verfolgen lässt, sei es „Germania Tod in Berlin“, „Leben Gundlings Friedrich von Preußen Lessings Schlaf Traum Schrei“ oder „Verkommenes Ufer Medeamaterial Landschaft mit Argonauten“31. Augenscheinlich ist noch eine weitere Tendenz: Hatte der junge Heiner Müller von Brecht das Konzept des politischen Theaters übernommen und ganz direkt Stellung

\footnotetext{
${ }^{24}$ Heiner Müller: Ein Brief (1975), in: ders.: Werke, Bd. 8: Schriften, 2005 (= W8), S. 174-177, Zitat S. 175.

${ }^{25}$ Müller: Traktor (Anm. 19).

${ }^{26}$ W4, S. $372 \mathrm{f}$.

${ }^{27} \mathrm{~W}$, S. $532 \mathrm{f}$.

${ }^{28}$ In: Platon, Sämtliche Werke, aus dem Griechischen von Friedrich Schleiermacher, hrsg. von Walter F. Otto, Ernesto Grassi und Gerd Plamböck, Bd. 2: Menon, Hippias I, Euthydemos, Menexenos, Kratylos, Lysis, Symposion, Hamburg 1957, S. 203-250, besonders S. 220-222.

${ }^{29}$ W4, S. $501 \mathrm{f}$.

${ }^{30}$ Siehe v.a. Walter Benjamin: Über den Begriff der Geschichte, in: ders.: Gesammelte Schriften, hrsg. von Rolf Tiedemann und Rolf Schweppenhäuser, Bd. I.2, Frankfurt a.M. 1974, S. 691-704.

${ }^{31}$ W5, S. $71-84$.
} 
zu brennenden Problemen der Gesellschaft bezogen, so sind konkrete politische Inhalte in den Stücken immer schwerer auszumachen. Gewalt ist nach wie vor ein zentrales Thema, erscheint aber mehr und mehr in der Form eines Kriegs der Geschlechter. Es ist, als zöge sich die Politik aus den Texten zurück, die immer disparater und hermetischer werden.

\section{Die Obsession des GANZEN: Bildbeschreibung als Circulus vitiosus}

Der Höhepunkt dieser Entwicklung ist mit Bildbeschreibung erreicht. Heiner Müller selbst spricht vom „Endpunkt oder Nullpunkt" einer ganzen Phase seiner schriftstellerischen Produktion. ${ }^{32}$ Allein schon die Zuordnung zu einer bestimmten Textsorte bereitet Schwierigkeiten. Dem Autor zufolge handelt es sich um ein Theaterstück, das auch 1985 von Ginka Tscholokowa uraufgeführt und seitdem immer wieder inszeniert wurde. In der vom Suhrkamp Verlag herausgegebenen Werkausgabe dagegen ist Bildbeschreibung unter den Prosawerken rubriziert. Dieses Vorgehen ist durchaus verständlich, handelt es sich doch um einen Prosatext, in dem ein ungenannt bleibendes Ich versucht, die Elemente eines Bildes beschreibend in einen Zusammenhang zu bringen. Ganz zu Anfang rückt kurz die Totale ins Bild, „eine Landschaft zwischen Steppe und Savanne, der Himmel preußisch blau“ ${ }^{\text {‘33. Dann }}$ schwenkt der Blick des Betrachters zu einzelnen Details: ein Baum, der sich „bei näherem Hinsehen“ (ebenda) als drei Bäume herausstellt, ein Haus, eine am unteren Bildrand befindliche Frau in einem Fellmantel, ein Mann, der im Begriff ist, aus dem Haus zu treten, vor dem Haus ein Tisch, darauf ein Glaspokal mit mehreren Früchten und ein umgestürztes, zerbrochenes Weinglas mit Resten einer dunklen Flüssigkeit, zwei Gartenstühle, von denen einer mit zerbrochener Lehne umgeworfen am Boden liegt, ein Vogel auf einem der Bäume, ein geöffnetes Fenster mit herauswehendem Vorhang, durch die Öffnung sichtbar ein an einer Innenwand aufgehängtes Vogelskelett. Diese Aufzählung ist jedoch irreführend, denn sie suggeriert Eindeutigkeit. In Wirklichkeit erweist sich die puzzleartige Zusammenfügung der verschiedenen Details zu einem Ganzen als unmöglich. Von Anfang an scheint der Betrachter seinen Augen nicht zu trauen, denn ständig berichtigt er das soeben noch Beschriebene. Konnte die präzisierende Korrektur der Anzahl der Bäume als Kamerafahrt des Blicks auf das Objekt hin verstanden werden, so zeigt sich schnell, dass es im Text von Modaladverbien wie „vielleicht“ und „wahrscheinlich“ und anderen Ausdrücken nur so wimmelt, die das Gesagte als zweifelhaft erscheinen lassen, wo-

\footnotetext{
${ }^{32}$ Heiner Müller: Solange wir an unsere Zukunft glauben, brauchen wir uns vor unserer Vergangenheit nicht zu fürchten, Gespräch mit Gregor Edelmann (1986), in: W10, S. 455-471, Zitat S. 457.

${ }^{33}$ W2, S. 112. Die folgenden Nachweise in Klammern im Text.
} 
bei allein die Konjunktion „oder“ siebenunddreißigmal verwendet wird. Ein gutes Beispiel hierfür ist die Beschreibung der Wolken am Himmel:

zwei riesige Wolken schwimmen darin, wie von Drahtskeletten zusammengehalten, jedenfalls von unbekannter Bauart, die linke größere könnte ein Gummitier aus einem Vergnügungspark sein, das sich von seiner Leine losgerissen hat, oder ein Stück Antarktis auf dem Heimflug (112). ${ }^{34}$

Darüber hinaus zeigt die Textstelle, wie die Beschreibung in Annahmen übergeht, die vom Bild selbst nicht bestätigt werden, Annahmen, die schließlich in Vorstellungen abgleiten, deren Ursprung nur im beschreibenden Ich selbst liegen kann. In dem Maße, wie das Bild Konturen annimmt, wird es von den Fantasien des Betrachters wieder zugedeckt. Die Beschreibung wird zur Be-schreibung, zur Übermalung; wobei der Begriff von Heiner Müller selbst eingeführt wird, der in einem Autorenkommentar Bildbeschreibung als eine „Übermalung der ALKESTIS“ (119) bezeichnet.

Als genauso prekär wie die Einfügung der Bildelemente in einen räumlichen Zusammenhang erweist sich der Versuch des Betrachters, interpretierend logische und chronologische Kohärenz herzustellen. Seine Bemühungen, dem Gesehenen einen Sinn zu verleihen, indem er es zu einer stringenten Geschichte zusammenführt, münden in Konstruktionen, die vom Bild nicht mehr gedeckt sind. So wird beispielsweise die Frau, deren Beine vom unteren Bildrand abgeschnitten sind, zunächst als aus der Erde Aufsteigende gedeutet, daraufhin als auferstehende Tote. Der Mann wird als potentieller Liebhaber und Mörder in diesen Zusammenhang eingepasst, der zerbrochene Stuhl schließlich dadurch erklärt, dass auf ihm „ein Mord vielleicht, oder ein wilder Geschlechtsakt, oder beides in einem“ (115) stattgefunden habe. Die Dynamisierung des ursprünglich unbewegten Bildes mündet dabei nicht nur in ein Szenario, sondern in verschiedene, einander widersprechende: Zunächst wird die Frau als Wiedergängerin aus dem Totenreich interpretiert, die ihren Mann besucht, um mit ihm zu schlafen, und von diesem erneut - „ICH HABE DIR GESAGT DU SOLLST NICHT WIEDERKOMMEN TOT IST TOT“ (118) - ermordet wird; der Vogel auf dem Baum erscheint dabei als Beobachter. In einer anderen Variante beißt die Frau dem Vogel den Kopf ab und gießt dessen Blut in das Glas auf dem Gartentisch. Und schließlich lenkt die Frau durch ihr Lachen den Mann von seinem mörderischen Vorhaben ab; der Vogel greift den Mann an und blendet ihn mit einem Schnabelhieb. Insgesamt ergibt sich ein Kreislauf der Gewalt, dessen Protagonisten, Mann, Frau und Vogel, abwechselnd die Position des Täters, des Opfers und des Beobachters einnehmen. In diesen Teufelskreis wird ebenfalls das beschreibende Ich hineingezogen: Ist es zunächst in der Rolle eines neutralen Betrachters, so entwickelt es durch den Kontakt mit dem Bild Gewaltphantasien, denen es zunehmend hilflos ausgeliefert ist und in denen es sich zu verlieren droht. So ist

\footnotetext{
${ }^{34}$ Hervorhebungen von Thomas Zenetti.
} 
verständlich, dass das Ich am Ende von Bildbeschreibung versucht, den Blick auf sich selbst zu richten:

an welchem Gerät ist die Linse befestigt, die dem Blick die Farben aussaugt, in welcher Augenhöhle ist die Netzhaut aufgespannt, wer ODER WAS fragt nach dem Bild, IM SPIEGEL WOHNEN, ist der Mann mit dem Tanzschritt ICH, mein Grab sein Gesicht, ICH die Frau mit der Wunde am Hals, rechts und links in Händen den geteilten Vogel, Blut am Mund, ICH der Vogel, der mit der Schrift seines Schnabels dem Mörder den Weg in die Nacht zeigt, ICH der gefrorene Sturm (119).

Die gestellte Frage indessen, wer nämlich hier überhaupt spricht, erfährt keine Antwort: Das Ich wird verfremdet durch die Setzung in Majuskeln, eine Schreibweise, die Heiner Müller auch in anderen Werken verwendet, um einen Textteil auszugliedern und als Fremdkörper, Zitat oder Selbstzitat mit meist obskurer Herkunft zu kennzeichnen. So verliert sich das Ich als „ICH“ sofort im Gesamtgewebe des Textes, einer Textur, die über Bildbeschreibung weit hinausreicht. Dennoch kann die beschreibende Instanz von ihrer Tätigkeit nicht ablassen; erscheinen doch ihre Bemühungen, die disparaten Bildelemente in ein „GANZE[S]“ (117) zu überführen, als die einzige Möglichkeit, sich ihrer Identität zu versichern. Die zunächst bizarr anmutende Fügung „IM SPIEGEL WOHNEN“ bestätigt diesen Befund. Das ICH reduziert sich auf Spiegelungen der verschiedenen Bildelemente, was der Infinitiv als subjektlose Konjugationsform noch unterstreicht. Mehr noch: Jacques Lacan hat dargelegt, wie sich das Ich kleiner Kinder durch das Betrachten ihres Spiegelbilds konstituiert, nämlich als Antizipation einer Einheit, die in diesem Stadium der Entwicklung real noch gar nicht besteht. ${ }^{35}$ Daraus folgt, so Lacan, ein lebenslanges Hinterherhinken des Individuums hinter ,seinem“ Bild. Das reflexive Ich, als kartesianisches „Cogito“ zum Angelpunkt der neuzeitlichen Philosophie erklärt, wird unversehens prekär und im wahrsten Sinne des Wortes imaginär. Hatte der Autor Alkestis ins Spiel gebracht, jene Königin, die sterben muss, weil ihr Gemahl zum Sterben nicht bereit ist, und die aus dem Totenreich zurückkehrt, so gerät auf einmal ein weiterer Mythos in den Blick: Ähnlich wie Sisyphos ist das ICH von Bildbeschreibung dazu verdammt, unaufhörlich das Bild zu durchstreifen, um sich als Ich zu konstituieren. Unaufhörlich, denn der gesamte Text besteht aus einem einzigen Satz, der kein Verweilen gestattet; unaufhörlich, denn am Ende löst sich das Ich als ICH wieder in die einzelnen Bildelemente auf und hat keine andere Wahl, als einen neuen Durchgang zu beginnen. Verzweifelt sucht es

die Lücke im Ablauf, das Andre in der Wiederkehr des Gleichen, das Stottern im sprachlosen Text, das Loch in der Ewigkeit, de[n] vielleicht erlösende[n] FEHLER (118).

\footnotetext{
${ }^{35}$ Jacques Lacan: Das Spiegelstadium als Bildner der Ichfunktion, in: ders.: Schriften I, ausgew. und hrsg. von Norbert Haas, Weinheim/Berlin 1986, S. 60-71; frz. Original: Le stade du miroir comme formateur de la fonction du Je (1936), in: Ecrits, Paris 1966, S. 93-100.
} 


\section{Unter preußisch blauem Himmel: \\ Verankerung von Bildbeschreibung in der deutschen Geschichte des zwanzigsten Jahrhunderts}

An diesem „eigentümliche[n] Text“, wie Hans-Thies Lehmann Bildbeschreibung apostrophiert ${ }^{36}$, beißt sich die Forschung seit nunmehr fast dreißig Jahren die Zähne aus. Immer wieder sind bestimmte Aspekte herausgearbeitet worden: die Problematik des $\mathrm{Ich}^{37}$, ikonographische Beziehungen zu René Magritte und anderen Malern $^{38}$, das Thema des Sehens und der Blendung ${ }^{39}$, die metapoetische Dimension $^{40}$, die zugrunde liegende Geschichtsauffassung. ${ }^{41}$ Die Verankerung von Bildbeschreibung in speziell der deutschen Geschichte und der Bezug zur Entstehungszeit des Texts sind jedoch bisher weitgehend vernachlässigt worden. ${ }^{42}$ In der Tat lässt sich lediglich ein Wort zu Beginn des Texts, nämlich „preußisch“, geographisch und politisch verorten. Christian Klein hat als erster hierauf hingewiesen und die ostdeutsche Heimat des Autors in ein geopolitisches Kräftefeld „zwischen Steppe und Savanne“, sprich der Sowjetunion als „Utopie von gestern“ und Afrika als „Utopie von morgen“ eingeordnet. ${ }^{43}$ Das Wort vom ,preußisch blauen Himmel“ transportiert

\footnotetext{
${ }^{36}$ Hans-Thies Lehmann: Theater der Blicke. Zu Heiner Müllers ,Bildbeschreibung', in: Dramatik der DDR, hrsg. von Ulrich Profitlich, Frankfurt a.M. 1987, S. 186-202, Zitat S. 186.

${ }^{37}$ Christian Klein: 'Paysage outre mort' ou l'implosion du sujet par l'écriture, in: Connaissance de la RDA, Nr. 26 (Juni 1988), S. 21-28.

${ }^{38}$ Marlies Janz: Der erblickte Blick. Kommentar zu Heiner Müllers ,Bildbeschreibung; in: Spiele und Spiegelungen von Schrecken und Tod. Zum Werk von Heiner Müller, Sonderband zum 60. Geburtstag des Dichters, hrsg. von Paul Gerhard Klussmann u. Heinrich Mohr, Bonn 1990, S. 173-188; Florian Vaßen, Images become Texts become Images: Heiner Müller's ,Bildbeschreibung '(Description of a Picture '), in: Heiner Müller. Contexts and History; A Collection of Essays from the Sydney German Studies Symposium 1994: Heiner Müller / Theatre-History-Performance, hrsg. von Gerhard Fischer, Tübingen 1995, S. 165-187.

${ }^{39}$ Lehmann: Theater der Blicke; Genia Schulz: Der zersetzte Blick. Sehzwang und Blendung bei Heiner Müller, in: Frank Hörnigk: Heiner Müller Material. Texte und Kommentare, Leipzig 1989, S. 165-182; Janz: Der erblickte Blick.

${ }^{40}$ Lehmann: Theater der Blicke; Klein: Paysage outre mort, Arlene Akiko Teraoka: Writing and Violence in Heiner Müller's «Bildbeschreibung», in: Sigrid Bauschinger / Susan L. Cocalis, hrsg. von Vom Wort zum Bild. Das neue Theater in Deutschland und den USA, Bern 1992, S. 179-198.

${ }^{41}$ Lehmann: Theater der Blicke; Ernst Grohotolsky: 'Der Engel der Geschichte'. Versuch über Heiner Müllers «Bildbeschreibung», in: Heinz Hartwig, hrsg. von Heiner Müller: Bildbeschreibung. Text und Materialien, Graz 1985, S. 27-29. Verwiesen sei auch auf Heiner Müllers ,Bildbeschreibung . Ende der Vorstellung, hrsg. von Ulrike Haß, Berlin 2005, $225 \mathrm{~S}$.

${ }^{42}$ Ich habe in einer 2007 veröffentlichten Arbeit versucht, die verschiedenen Dimensionen von Bildbeschreibung im Begriff des „strukturell politischen Texts“ zusammenzuführen (Thomas Zenetti: Du texte-hydre au texte-sphinx. Les inserts dans le théâtre de Heiner Müller, Bern 2007, S. 193-326). Der vorliegende Artikel konzentriert sich auf den Aspekt der Situation in Deutschland gegen Ende des Kalten Kriegs.

${ }^{43}$ Klein: Paysage outre mort, S. 23. Siehe auch Axel Schalk, der Bildbeschreibung in Beziehung zur nuklearen Aufrüstung durch die beiden Militärblöcke in den 1980er Jahren setzt. Seiner Grundthese
} 
indessen weitere Konnotationen: Als hervorragender Kenner des Werks von Bertolt Brecht kannte Heiner Müller mit Sicherheit dessen Gedicht über Kleists Prinzen von Homburg, der nach seiner Rettung als ,in preußisch blauer Nacht“ auf dem Rücken liegender „Ausbund von Kriegerstolz und Knechtsverstand“ gekennzeichnet wird. ${ }^{44}$ So leitet das preußische Blau zum Themenkomplex der „deutschen Misere“ über, den Müller besonders in Germania Tod und Leben Gundlings gestaltet hat. Berücksichtigt man weiterhin, dass Preußischblau ein Eisenzyanidpigment ist, das Blausäure freisetzen kann, und Blausäure wiederum unter dem Namen „Zyklon B“ in den Gaskammern der Nationalsozialisten eingesetzt wurde, dann scheint auch für Bildbeschreibung zu gelten, was Heiner Müller bereits in einem seiner frühesten Gedichte geschrieben hatte: „DER TERROR VON DEM ICH SCHREIBE KOMMT AUS DEUTSCHLAND““. ${ }^{45}$ So genügt ein einziges Wort, um eine Suche in Gang zu setzen, auf der der Interpret den Text erneut durchstreift - hierin durchaus ähnlich dem beschreibenden Ich, das von Bildelement zu Bildelement irrt -, um eine Bestätigung für seine Hypothese zu finden. Und tatsächlich verweist die aus dem Fenster wehende Gardine auf einen Sturm, der zu der tödlichen Chemikalie in direkter Beziehung steht:

der Sturm scheint aus dem Haus zu kommen, in den Bäumen keine Spur von Wind, oder zieht die Frau den Sturm an, oder ruft ihn hervor mit ihre Erscheinung, der auf sie gewartet hat in der Asche des Kamins, was oder wer ist verbrannt worden, ein Kind, eine andere Frau, ein Geliebter (114).

Weiteres Nachforschen im Text legt eine Beziehung zu Paul Celans Todesfuge frei, der vielleicht berühmtesten literarischen Gestaltung der Shoa. Der aus dem Haus tretende Mann in seiner Doppelrolle als Liebender und Mörder, nicht weniger als das „,blond[e] oder weißgrau[e]“ (113) Haar der Frau, verweisen auf Motive in Celans Gedicht. ${ }^{46}$ Nicht zuletzt aber erinnert Bildbeschreibung durch seine atemlose Parataxe an Todesfuge, die ohne jede Interpunktion zwei Grundthemen, deutsche Kultur und deutsche Barbarei, einander verfolgen und umspielen lässt. Beschreibt Heiner Müller einen „Terror“, der ,aus Deutschland“ kommt, so ist dies auch ein Nachhall von Celans Wort: „Der Tod ist ein Meister aus Deutschland“ ${ }^{47}$

jedoch kann nicht zugestimmt werden, die Inkommensurabilität der atomaren Bedrohung schlage sich in einem Text nieder, der sich absichtlich jedem Verständnis entziehe (Axel Schalk: Als Galilei abschwor. Bertolt Brecht und Heiner Müller und die Bombe, in: Ian Wallace, Denis Tate, Gerd Labroisse: Heiner Müller: Probleme und Perspektiven, Amsterdam/Atlanta 2000, S. 153-170).

${ }^{44}$ Bertolt Brecht: Über Kleists Stück ,Der Prinz von Homburg ' (1939), in: ders.: Werke. Große kommentierte Berliner und Frankfurter Ausgabe, Berlin/Weimar/Frankfurt a.M. (= GBFA), Bd. 10: Gedichte 1, 1988, S. 272f.

${ }^{45}$ Heiner Müller: Werke, Bd. 1: Die Gedichte, 1998 (=W1), S. 8; das Gedicht stammt aus den frühen 1950er Jahren.

${ }^{46}$ Paul Celan: Todesfuge (1944-45), in: ders.: Gesammelte Werke in fünf Bänden, Frankfurt a.M. 1983, Bd. I: Gedichte I, S. 39-42.

${ }^{47}$ Ebd. 
Bildbeschreibung bezieht sich aber nicht nur auf das Grauen der Judenvernichtung, das unauflöslich mit der deutschen Geschichte verknüpft ist, sondern spielt auch auf die Problematik revolutionärer Gewalt an, die Menschen tötet, um eine menschliche Gesellschaft durchzusetzen. Wie ein Leitmotiv hatte dieses Paradox bereits Heiner Müllers Stück Mauser durchzogen:

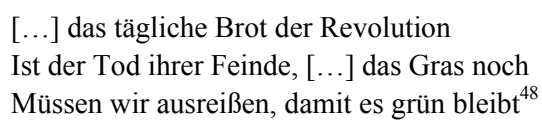

Wenn nun der Autor dieses Motiv in der Variante ,überflüssig das Gras auszureißen, die SONNE, vielleicht eine Vielzahl von SONNEN, verbrennt es“ (116) verwendet, so bedeutet dies nicht weniger als eine Abkehr von der Hoffnung, revolutionäre Gewalt könnte der Spirale von Gewalt und Gegengewalt ein Ende setzen, die sich durch die Geschichte zieht. Hans-Thies Lehmann hat das "harte Licht“ (113) der „Vielzahl von SONNEN“ mit dem Blitz der Atombombe identifiziert und in Verbindung zum Titel von Robert Jungks Buch über Hiroshima gebracht. ${ }^{49}$ In der ersten Hälfte der 1980er Jahre wird in Folge der atomaren Aufrüstung auf dem Gebiet beider deutscher Staaten die steigende Bedrohung in breiten Schichten der Bevölkerung diskutiert. Auf einem Gesprächsforum in Ostberlin drückt Heiner Müller eine Befürchtung aus, die sich wie eine Antwort liest auf die in Mauser formulierte Alternative „die Revolution wird siegen oder der Mensch wird nicht sein / Sondern verschwinden in zunehmender Menschheit ${ }^{\text {‘50 }}$

Hinter der Frage Krieg oder Frieden steht mit der nuklearen Bedrohung die schrecklichere Frage, ob noch ein anderer Frieden denkbar ist als der Frieden der Ausbeutung und der Korruption. Der Alptraum, dass die Alternative Sozialismus oder Barbarei abgelöst wird durch die Alternative Untergang oder Barbarei. Das Ende der Menschheit als Preis für das Überleben des Planeten. ${ }^{51}$

\section{Zwischen Posthistoire und Ausbruch aus dem Teufelskreis: Bildbeschreibung als Geschichtsbild-Beschreibung}

Bildbeschreibung setzt die alptraumhafte Alternative Ende mit Schrecken oder Schrecken ohne Ende in Szene. Das sich zwanghaft aufdrängende Bild von der „Blutpumpe des täglichen Mords, Mann gegen Vogel und Frau, Frau gegen Vogel und Mann, Vogel gegen Frau und Mann“ (118) löst eine verzweifelte Suche des

\footnotetext{
${ }^{48}$ Heiner Müller: Mauser; insgesamt erscheint das Zitat achtmal im Text.

${ }^{49}$ Lehmann: Theater der Blicke, S. 194; bei dem angeführten Buch handelt es sich um Robert Jungk: Heller als tausend Sonnen: Das Schicksal der Atomforscher, Stuttgart 1956.

${ }^{50}$ W4, S. 254.

${ }^{51}$ Heiner Müller: Diskussionsbeitrag auf der „Berliner Begegnung“ vom 13. und 14. Dezember 1981, in: W8, S. 247-248, Zitat S. 247.
} 
beschreibenden Ich nach einem Ausweg, nach dem „Andre[n] in der Wiederkehr des Gleichen" (ebd.) aus. Nicht zufällig erinnert das Zitat an Friedrich Nietzsche, durch dessen Werk dieser „Gedanke[...] der Gedanken“汭 geistert, gefasst als „,ewige Wiederkehr", häufiger noch in der Variante „ewige Wiederkunft". Wobei sich die Festlegung auf eine präzise Bedeutung als schwierig erweist: Im Lenzer Heide Fragment $^{53}$ etwa meint der Philosoph damit das Schreckgespenst einer endlosen Wiederholung des Identischen, an anderer Stelle das durchaus positiv verstandene Ausbrechen aus der neuzeitlichen Vorstellung von einem linearen Fortschreiten der Geschichte. ${ }^{54}$ Der junge Heiner Müller hat direkt nach Kriegsende intensiv Nietzsche gelesen, als „Gegengift gegen die Nivellierungstendenzen“ in der SBZ, wie er sagt $^{55}$, und, so kann man ergänzen, ganz allgemein gegen die Fortschrittsgläubigkeit des orthodoxen Marxismus. Wenn er später immer wieder die große Bedeutung Friedrich Nietzsches für die eigene literarische Produktion betont ${ }^{56}$, so bedeutet dies nicht, dass er sich dessen Gedankenwelt systematisch angeeignet hätte - ganz im Gegenteil, es ist gerade das Antisystematische, das „nomadische Denken “57 des Philosophen, das ihn anzieht. Dementsprechend macht er einzelne Gedanken Nietzsches für sich fruchtbar, indem er sie mit eigenen verbindet; aber eben nicht im Sinne eines postmodernen Hantierens mit Versatzstücken der Vergangenheit, sondern als Versuch, jenseits der traditionellen Systeme einen neuen Ansatz zu finden. Und so interessiert ihn auch nicht eigentlich

die Wiederkehr des Gleichen, sondern unter ganz anderen Umständen die Wiederkehr des Gleichen als eines Anderen. Das wäre eine Differenz. Mein Interesse an der Wiederkehr des Gleichen ist ein Interesse an der Sprengung des Kontinuums, auch an Literatur als Sprengsatz und Potential von Revolution. $^{58}$

Genau diese Hoffnung auf Sprengung des Kontinuums hatte die Theorie der Posthistoire inzwischen aufgegeben. Bereits in den 1960er Jahren hatte der deutsche

\footnotetext{
${ }^{52}$ In: Friedrich Nietzsche: Sämtliche Werke. Kritische Studienausgabe in 15 Bänden, hrsg. von Giorgio Colli und Mazzino Montinari, München 1980 (= KSA), Bd. 9: Nachgelassene Fragmente 1880-1882, S. 496.

${ }^{53}$ Friedrich Nietzsche: Der europäische Nihilismus, in: KSA, Bd. 12: Nachgelassene Fragmente 1885-1887, S. 211-217, insbesondere S. 213.

${ }^{54}$ Friedrich Nietzsche: Also sprach Zarathustra (= KSA, Bd. 4), S. $272 \mathrm{f}$. Zu den verschiedenen Implikationen des Begriffs siehe den Artikel „Ewige Wiederkunft“ von Miguel Skirl, in: NietzscheHandbuch. Leben, Werk, Wirkung, hrsg. von Henning Ottmann, Stuttgart/Weimar 2000, S. 222-230.

${ }^{55}$ Heiner Müller: Geschichtspessimismus oder Geschichtsoptimismus, das sind nur zwei Begriffe für Geschichtsunkenntnis, Gespräch mit Walter Höllerer (1987), in: W10, S. 522-579, Zitat S. 535.

${ }^{56}$ Siehe z.B. Heiner Müller: Was gebraucht wird: mehr Utopie, mehr Phantasie und mehr Freiräume für Phantasie, Gespräch mit Ulrich Dietzel (1984), in: W10, 2008, S. 318-345; Heiner Müller: Pflugschar des Bösen, Gespräch mit Alexander Kluge (1992), in: W12, S. 293-300.

${ }^{57}$ Müller: Was gebraucht wird, S. 336.

${ }^{58}$ Ebd., S. 335.
} 
Anthropologe Arnold Gehlen das „Ende der Philosophie“699 verkündet: Westlicher Kapitalismus und real existierender Sozialismus hätten sich zu Systemen verfestigt, die zwar noch Ausdifferenzierung, aber keine wirkliche Veränderung mehr erlaubten. War in diesem Zusammenhang zum ersten Mal der Begriff des „Posthistoire“ verwendet worden $^{60}$, so wird dieser rund zwanzig Jahre später von zwei französischen Philosophen wenngleich nicht direkt zitiert, so doch inhaltlich näher bestimmt. Komplementär zu Gehlen, der vorrangig das Stocken des geschichtlichen Prozesses postuliert hatte, setzt der Poststrukturalist Jean Baudrillard den Akzent auf die allmähliche Abkoppelung der Zeichen von der geschichtlichen, politischen und ökonomischen Wirklichkeit. ${ }^{61}$ Baudrillard zufolge befinden wir uns am Beginn einer „Ära der Simulation“62, in der die verschiedenen Simulakra in einem gigantischen Kreislauf aufeinander verweisen, ohne sich noch auf die Realität zu beziehen. Derlei Bewegung ohne wirklichen Fortschritt fasst der Medientheoretiker Paul Virilio im Begriff des „rasenden Stillstands“63: Der unsere Zivilisation beherrschende Geschwindigkeitswahn lasse Raum und Zeit implodieren und mache politisches Handeln tendenziell unmöglich. So unterschiedlich diese Theorien im Einzelnen auch sein mögen, verabschieden sie doch sämtlich die Vorstellung von Geschichte als einem linearen Prozess und vom Menschen als dessen steuerndem Subjekt. Heiner Müller kennt diese Ansätze, bezieht sich auch auf Baudrillard ${ }^{64}$ und Virilio ${ }^{65}$, grenzt sich aber insgesamt mit Vehemenz vom Begriff des Posthistoire ab:

Wie schamlos die Lüge vom POSTHISTOIRE vor der barbarischen Wirklichkeit unserer Vorgeschichte. [...] Nicht eh Geschichte passiert ist, lohnt der gemeinsame Untergang im Frost der Entropie, oder, politisch verkürzt, im Atomblitz, der das Ende der Utopien und der Beginn einer Wirklichkeit jenseits des Menschen sein wird. ${ }^{66}$

${ }^{59}$ Arnold Gehlen: Über kulturelle Kristallisation, in: Studien zur Anthropologie und Soziologie, Neuwied/Berlin 1963, S. 311-338, Zitat S. 316.

${ }^{60}$ Ebd., S. 323.

${ }^{61}$ Jean Baudrillard: Die Präzession der Simulakra, in: Agonie des Realen, aus dem Französischen von Lothar Kurzawa und Volker Schaefer, Berlin (West) 1978, S. 7-69; frz. Original: La précession des simulacres (1978), in: Simulacres et simulation, Paris 1981, S. 9-68.

${ }^{62}$ Baudrillard: Die Präzession der Simulakra, S. 9; frz. Original: La précession des simulacres, S. 11.

${ }^{63}$ Paul Virilio: Rasender Stillstand, aus dem Französischen von Bernd Wilczek, München 1992; frz. Original: L'inertie polaire, Paris 1990. Seine Grundgedanken veröffentlichte Virilio jedoch bereits 1977 unter dem Titel Vitesse et Politique. Essai de dromologie, in deutscher Übersetzung unter dem Titel Geschwindigkeit und Politik 1980 im Merve-Verlag.

${ }^{64}$ Siehe insbesondere Heiner Müller: Ich glaube an Konflikt, sonst glaube ich an nichts, Gespräch mit Sylvère Lothringer (1982), in: W10, S. 175-223, Zitat S. 179f.

${ }^{65}$ Siehe insbesondere den von Virilio stammenden Ansatz der Beschleunigung als Grundmuster unserer Zivilisation in Heiner Müller: Da trinke ich lieber Benzin zum Frühstück, Gespräch mit Frank M. Raddatz (1989), in: W11, S. 431-445, Zitat S. 436f.

${ }^{66}$ Heiner Müller: Die Wunde Woyzeck [Rede zur Verleihung des Büchner-Preises 1985], in: W8, S. 281-283, Zitat S. $282 f$. 
Völlig verständlich werden diese wütenden Angriffe erst Jahre später, als Francis Fukuyama in seinem Buch The End of History behauptet, die Geschichte der Menschheit sei nach dem Zusammenbruch des „real existierenden Sozialismus“ in der liberalistischen Demokratie amerikanischer Prägung zu ihrem definitiven Ende gekommen. ${ }^{67}$

Literatur leistet jedoch mehr als nur die Illustrierung von Theorie durch sprachliche Bilder. „Wenn man eine Idee in ein Bild übersetzt“, sagt Heiner Müller, „wird entweder das Bild schief oder die Idee explodiert. Ich bin mehr für die Explosion. “68 In Bildbeschreibung erscheint diese Explosion als

die eine unaufhörliche Bewegung [...], die den Rahmen sprengt, der Flug, das Triebwerk der Wurzeln Erdbrocken und Grundwasser regnend, sichtbar zwischen Blick und Blick, wenn das Auge ALLES GESEHN sich blinzelnd über dem Bild schließt (113).

Nicht zufällig wird die aus dem Boden wachsende Frau zum Ausgangspunkt dieser Bewegung. Sie, die im Text insgesamt dreimal als „Engel“ bezeichnet wird, weist Parallelen zu einer Figur auf, die Müller als ,glücklosen Engel“ in seine Bearbeitung von Brechts unvollendetem Glücksgott-Projekt ${ }^{69}$ eingefügt hatte:

DER GLÜCKLOSE ENGEL. Hinter ihm schwemmt Vergangenheit an, schüttet Geröll auf Flügel und Schultern, mit Lärm wie von begrabnen Trommeln, während vor ihm sich die Zukunft staut, seine Augen eindrückt, die Augäpfel sprengt wie ein Stern, das Wort umdreht zum tönenden Knebel, ihn würgt mit seinem Atem. Eine Zeitlang sieht man noch sein Flügelschlagen, hört in das Rauschen die Steinschläge vor über hinter ihm niedergehn, lauter je heftiger die vergebliche Bewegung, vereinzelt, wenn sie langsamer wird. Dann schließt sich über ihm der Augenblick: auf dem schnell verschütteten Stehplatz kommt der glücklose Engel zur Ruhe, wartend auf Geschichte in der Versteinerung von Flug Blick Atem. Bis das erneute Rauschen mächtiger Flügelschläge sich in Wellen durch den Stein fortpflanzt und seinen Flug anzeigt. ${ }^{70}$

Bereits in diesem frühen Text Heiner Müllers wird das Stillstellen der historischen Situation beschrieben, aber auch die Hoffnung auf ein neuerliches InBewegung-Kommen des Geschichtsprozesses. Die Frau in Bildbeschreibung, die „Wurzeln Erdbrocken und Grundwasser regnend“ der Erde entsteigt, gleicht dem Engel, der flügelrauschend der Versteinerung entkommt. Immer wieder ist auf die Beziehung von Bildbeschreibung zu einem anderen „Engel der Geschichte“ hinge-

\footnotetext{
${ }^{67}$ Francis Fukuyama: The End of History and the Last Man, New York 1992; deutsche Ausgabe: Das Ende der Geschichte. Wo stehen wir?, aus dem Amerikanischen von Helmut Dierlamm, Ute Mihr und Karlheinz Dürr, München 1992. Zuvor hatte Fukuyama seine Thesen in dem Artikel The End of History?, in: The National Interest, Nr. 15, Sommer 1989, veröffentlicht.

${ }^{68}$ Heiner Müller: Was ein Kunstwerk kann, ist Sehnsucht wecken nach einem anderen Zustand der Welt, Gespräch mit Urs Jenny und Hellmuth Karasek (1983), in: W10, S. 266-279, Zitat S. 270.

${ }^{69}$ Bertolt Brecht: Die Reisen des Glücksgotts, in: GBFA, Bd. 10: Stücke 10, 1997, S. 922-937.

${ }^{70}$ Heiner Müller: Der glücklose Engel, in: W1, S. 53; eingefügt in Heiner Müller: Glücksgott (1958), in: W3, S. 163-180, Zitat S. 180.
} 
wiesen worden ${ }^{71}$, der emblematischen Figur in der neunten von Walter Benjamins Thesen über den Begriff der Geschichte. ${ }^{72}$ Benjamins Engel starrt gebannt auf eine Vergangenheit, die unablässig Trümmer vor seinen Füßen aufhäuft. Er möchte das Zerschlagene zusammenfügen, wird aber vom Sturm, der vom Paradies her weht, rückwärts in die Zukunft getrieben. Auch bei diesem Text handelt es sich um eine Bildbeschreibung, verwendet Benjamin doch eine Zeichnung Paul Klees ${ }^{73}$ als Vorlage, die er allerdings mit eigenen Vorstellungen regelrecht übermalt, um seine Konzeption von Geschichte als einer Anhäufung von Katastrophen zu illustrieren. Trotz vielfältiger intertextueller und ikonographischer Bezüge hebt sich Bildbeschreibung dadurch heraus, dass hier ein Rest Hoffnung auf einen Ausstieg aus dem Teufelskreis von Katastrophen bleibt. Verbunden ist diese Hoffnung allerdings mit der Angst, das Kairos zu verpassen, weil der ,erlösende FEHLER“ (118) „,während des Blinzelns passiert, der Sehschlitz in die Zeit sich auftut zwischen Blick und Blick“ (119). Dies verbindet Müllers Text mit einer enigmatischen Geschichte, die Franz Kafka 1914 in seinem Tagebuch notiert hat: Der Erzähler wartet in seinem Zimmer auf eine Begegnung, von der er sich Befreiung erhofft. Was jedoch durch die Decke bricht und zunächst wie ein Engel aussieht, stellt sich, nachdem der Protagonist den einen Augenblick lang gesenkten Blick wieder hebt, als bemalte Holzfigur heraus. ${ }^{74}$

Kafkas Tagebucheintrag war unmittelbar vor Ausbruch des Ersten Weltkriegs entstanden, Paul Klees Zeichnung kurz nach dessen Ende, Benjamins Text mitten im Zweiten Weltkrieg und Müllers Glückloser Engel in einer Periode stalinistischer Verhärtung. ${ }^{75}$ Die Abfassung von Bildbeschreibung fällt in eine Zeit, die sich im Zeichen atomarer Nachrüstung vor eine Alternative gestellt sieht, die Müller an anderer Stelle eindringlich beschrieben hat: ein Ende mit Schrecken, oder aber ein Gleichgewicht des Schreckens, das gesellschaftlichen Fortschritt in beiden Lagern unmöglich zu machen droht. 1984 ist jedoch nicht nur die Jahreszahl der „schönen neuen Welt", sondern auch der historische Moment, in dem die Entscheidung ansteht, wer in der Sowjetunion den schwerkranken Tschernjenko in der Macht beerben soll: der Hardliner Romanow oder der Reformer Gorbatschow. Das „Stück Antarktis auf dem Heimflug" verweist auf ein mögliches Tauwetter nach vier Jahrzehnten Kalten Kriegs, skizziert aber auch ein neues geopolitisches Spannungsfeld zwischen Nord und Süd, als Alternative zum Antagonismus zwischen Erster und Zweiter Welt. Geschichte kommt möglicherweise wieder in Gang, aber mit ungewissem Ausgang. Die Umsetzung des marxistischen Grundgedankens, dass der

\footnotetext{
${ }^{71}$ Als erster Grohotolsky: Der Engel der Geschichte.

${ }^{72}$ Walter Benjamin: Über den Begriff der Geschichte [IX], S. 697f.

${ }^{73}$ Paul Klee: Angelus Novus (1920), The Israel Museum, Jerusalem.

${ }^{74}$ In: Franz Kafka: Tagebücher, hrsg. von Hans-Gerd Koch, Michael Müller und Malcolm Pasley, Frankfurt a.M. 1990, S. 538-541.

${ }^{75} \mathrm{Zu}$ den Beziehungen der verschiedenen Engelsdarstellungen zu Bildbeschreibung siehe das Kapitel Un ange peut en cacher (en ,recouvrir') d'autres: Benjamin, Klee, Kafka, Müller, in: Thomas Zenetti: Du texte-hydre au texte-sphinx, S. 298-316.
} 
Mensch zum Menschen wird, indem er sich als Subjekt der Geschichte konstituiert, wird in Müllers Text als verzweifelter Versuch vorgeführt, keineswegs jedoch als gelungen postuliert. Ein Vierteljahrhundert nach dem Fall der Mauer scheint Francis Fukuyama Recht behalten zu haben. Heiner Müller ist 1995 gestorben; was von ihm bleibt, sind „einsame Texte, die auf Geschichte warten“. ${ }^{76}$

\section{Literatur}

Baudrillard, Jean: Die Präzession der Simulakra, in: Agonie des Realen, aus dem Französischen von Lothar Kurzawa und Volker Schaefer, Berlin 1978, S. 7-69; frz. Originalausgabe: La précession des simulacres (1978), in: Simulacres et simulation, Paris 1981, S. 9-68.

Benjamin, Walter: Über den Begriff der Geschichte, in: Gesammelte Schriften, hrsg. von Rolf Tiedemann und Rolf Schweppenhäuser, Band I.2., Frankfurt a.M. 1974, S. 691-704.

Brecht, Bertolt: Werke. Große kommentierte Berliner und Frankfurter Ausgabe, Berlin/Weimar/Frankfurt a.M. $1988 \mathrm{ff}$.

Celan, Paul: Todesfuge (1944-45), in: Gesammelte Werke in fünf Bänden, Band I, Frankfurt a.M. 1983, Gedichte I, S. 39-42.

Fukuyama, Francis: Das Ende der Geschichte. Wo stehen wir?, aus dem Amerikanischen von Helmut Dierlamm, Ute Mihr und Karlheinz Dürr, München 1992; amerikanische Originalausgabe: The End of History and the Last Man, New York 1992.

Gehlen, Arnold: Über kulturelle Kristallisation, in: Studien zur Anthropologie und Soziologie, Neuwied/Berlin 1963, S. 311-338.

Grohotolsky, Ernst: ,Der Engel der Geschichte'. Versuch über Heiner Müllers ,Bildbeschreibung', in: Heiner Müller: Bildbeschreibung. Text und Materialien, hrsg. von Heinz Hartwig, Graz 1985, S. 27-29.

„Die Hamletmaschine“. Heiner Müllers Endspiel, hrsg. von Theo Girshausen, Köln 1978.

Heiner Müllers ,Bildbeschreibung ‘. Ende der Vorstellung, hrsg. von Ulrike Haß, Berlin 2005.

Janz, Marlies: Der erblickte Blick. Kommentar zu Heiner Müllers ,Bildbeschreibung', in: Spiele und Spiegelungen von Schrecken und Tod. Zum Werk von Heiner Müller. Sonderband zum 60. Geburtstag des Dichters, hrsg. von Paul Gerhard Klussmann u. Heinrich Mohr, Bonn 1990, S. 173-188.

Kafka, Franz: Tagebücher, hrsg. von Hans-Gerd Koch, Michael Müller und Malcolm Pasley, Frankfurt a.M. 1990.

Klein, Christian: ,Paysage outre mort' ou l'implosion du sujet par l'écriture, in: Connaissance de la $R D A$, Nr. 26 (Juni 1988), S. 21-28.

Lacan, Jacques: Schriften, ausgew. und hrsg. von Norbert Haas, Weinheim/Berlin 1986; frz. Originalausgabe: Ecrits, Paris 1966.

Lehmann, Hans-Thies: Theater der Blicke. Zu Heiner Müllers ,Bildbeschreibung', in: Dramatik der $D D R$, hrsg. von Ulrich Profitlich, Frankfurt a.M. 1987, S. 186-202.

Müller, Heiner: Werke, Frankfurt a.M. 1998.

Müller, Heiner: Manuscrits de Hamlet-Machine, transcription de Julia Bernhard, traduction de Jean Jourdheuil et Heinz Schwarzinger, Paris 2003.

Nietzsche, Friedrich: Sämtliche Werke. Kritische Studienausgabe in 15 Bänden, hrsg. von Giorgio Colli und Mazzino Montinari, München 1980.

\footnotetext{
${ }^{76}$ Heiner Müller: Verabschiedung des Lehrstücks (1977), in: W8, S. 187.
} 
Platon: Symposion, in: Sämtliche Werke, aus dem Griechischen von Friedrich Schleiermacher, hrsg. von Walter F. Otto, Ernesto Grassi und Gerd Plamböck, Band 2: Menon, Hippias I, Euthydemos, Menexenos, Kratylos, Lysis, Symposion, Hamburg 1957, S. 203-250.

Schalk, Axel: Als Galilei abschwor. Bertolt Brecht und Heiner Müller und die Bombe, in: Heiner Müller: Probleme und Perspektiven, hrsg. von Ian Wallace, Dennis Tate und Gerd Labroisse, Amsterdam/Atlanta 2000, S. 153-170.

Schulz, Genia: Der zersetzte Blick. Sehzwang und Blendung bei Heiner Müller, in: Frank Hörnigk: Heiner Müller Material. Texte und Kommentare, Leipzig 1989, S. 165-182.

Skirl, Miguel: Ewige Wiederkunft, in: Nietzsche-Handbuch. Leben, Werk, Wirkung, hrsg. von Henning Ottmann, Stuttgart/Weimar 2000, S. 222-230.

Teraoka, Arlene Akiko: The Silence of Entropy or Universal Discourse. The Postmodernist Poetics of Heiner Müller, New York/Bern/Frankfurt a.M. 1985.

Teraoka, Arlene Akiko: Writing and Violence in Heiner Müller's ,Bildbeschreibung ', in: Vom Wort zum Bild. Das neue Theater in Deutschland und den USA, hrsg. von Sigrid Bauschinger u. Susan L. Cocalis, Bern 1992, S. 179-198.

Vaßen, Florian: Images become Texts become Images: Heiner Müller's ,Bildbeschreibung ' (,Description of a Picture"), in: Heiner Müller. Contexts and History. A Collection of Essays from the Sydney German Studies Symposium 1994: Heiner Müller. Theatre-History-Performance, hrsg. von Gerhard Fischer, Tübingen 1995, S. 165-187.

Virilio, Paul: Geschwindigkeit und Politik, aus dem Französischen von Ronald Voullié, Berlin (West) 1980; frz. Originalausgabe: Vitesse et Politique. Essai de dromologie, Paris 1977.

Virilio, Paul: Rasender Stillstand, aus dem Französischen von Bernd Wilczek, München 1992; frz. Originalausgabe: L'inertie polaire, Paris 1990.

Zenetti, Thomas: Du texte-hydre au texte-sphinx. Les inserts dans le théâtre de Heiner Müller, Bern 2007. 